\begin{tabular}{|c|l|}
\hline Title & Interaction between viral RNA silencing suppressors and host factors in plant immunity \\
\hline Author(s) & Nakahara, Kenji S; Masuta, Chikara \\
\hline Citation & $\begin{array}{l}\text { Current Opinion in Plant Biology, 20,88-95 } \\
\text { https://doi.org/10.1016/.pbi.2014.05.004 }\end{array}$ \\
\hline Issue Date & 2014.04 \\
\hline Doc URL & http://hdl.handle.net/2115/56348 \\
\hline Type & article (author version) \\
\hline File Information & text.pdf \\
\hline
\end{tabular}

Instructions for use 


\section{Interaction between viral RNA silencing suppressors and host factors}

2 in plant immunity

3 Kenji S Nakahara and Chikara Masuta

4

5 Short title

6 Zigzag model of the arms race between plants and viruses

7

\section{Address}

9 Plant Breeding Science, Research Faculty of Agriculture, Hokkaido University,

10 Sapporo, 060-8589, Japan

12 Corresponding authors: Masuta, Chikara (masuta@res.agr.hokudai.ac.jp) and

$13 \quad$ Nakahara, Kenji S (knakahar@res.agr.hokudai.ac.jp)

15 To elucidate events in the molecular arms race between the host and pathogen in

16 evaluating plant immunity, a zigzag model is useful for uncovering aspects common to

17 different host-pathogen interactions. By analogy of the steps in virus-host interactions

18 with the steps in the standard zigzag model outlined in recent papers, we may regard

19 RNA silencing as pattern-triggered immunity (PTI) against viruses, RNA silencing

20 suppressors (RSSs) as effectors to overcome host RNA silencing and resistance gene

21 (R-gene)-mediated defense as effector-triggered immunity (ETI) recognizing RSSs as

22 avirulence proteins. However, because the standard zigzag model does not fully apply

23 to some unique aspects in the interactions between a plant host and virus, we here

24 defined a model especially designed for viruses. Although we simplified the

25 phenomena involved in the virus-host interactions in the model, certain specific

26 interactive steps can be explained by integrating additional host factors into the model.

27 These host factors are thought to play an important role in maintaining the efficacy of 
the various steps in the main pathway of defense against viruses in this model for virus-plant interactions. For example, we propose candidates that may interact with viral RSSs to induce the resistance response.

\section{Introduction}

Plants use two major strategies to defend against pathogens; the resistance (R)-proteinmediated strategy works effectively against diverse pathogens, including fungi, bacteria and viruses, while the RNA silencing strategy is a major antiviral mechanism [1-3]. Most viruses encode RNA silencing suppressors (RSSs) to interfere with RNA silencing $[4,5]$. As a consequence of the particular strategy used in the battle between virus and host, infected plants develop various symptoms [6]. According to the zigzag model (Figure 1A) to explain the two-branched immune system of plants in response to a plant pathogen $[7 \cdot, 8]$, R-protein-mediated resistance developed to control a pathogen that had overcome basal resistance or innate immunity, the first line of preformed, inducible defenses against the major groups of pathogens. Basal resistance starts with the detection of pathogen-associated molecular patterns (PAMPs), such as bacterial flagellin and fungal chitin, by the host's patternrecognition receptors (PRRs). In the zigzag model, it is defined as pattern-triggered immunity (PTI). PRRs for bacteria and fungi have been identified, and these are mostly receptor-like kinases, which were once classified in the R-protein family. For example, the host transmembrane FLS2 protein recognizes the flg22 peptide from Pseudomonas flagellin [9]. To circumvent basal defense (PTI), pathogens produce

50 effector proteins. When pathogen effectors overcame PTI, plants next evolutionally developed R-proteins to activate effector-triggered immunity (ETI), by which host proteins recognize the effectors as avirulence (Avr) factors, which then induces an amplified version of resistance comparable to PTI. R-protein-mediated resistance is often accompanied by a hypersensitive response (HR), which is observed as local 
necrotic lesions. Therefore, we regard the HR-associated resistance response as a consequence of R-protein-mediated resistance, unless the HR pathway is independent of this resistance pathway.

There are not many comparative studies between antiviral and antibacterial/antifungal immune responses. Mandadi and Scholthof [10] have once reviewed analogous viral and nonviral immune concepts, but it was found not to be so simple to define viral PTI, ETI and ETS finding concrete examples; they did not actually integrate RNA silencing into their model. On the other hand, because RNA silencing against viruses is reminiscent of basal resistance against fungi and bacteria, by regarding RNA silencing as a type of PTI, viruses can be also integrated in a modified zigzag model; here, viral double-stranded RNA (dsRNA) corresponds to a PAMP [11,12]. However, there are certainly differences between viruses and other pathogens in their molecular interactions with plant hosts. In devising a model for viruses, we here integrate additional host factors to explain certain virus-host interactions and highlight aspects of the anti-viral defense that differ from the standard zigzag model for fungi and bacteria. In addition, we focus on the molecular cross-talk between RNA silencing and R-protein-mediated resistance. Figure $1 \mathrm{C}$ shows our entire scheme to explain the hostvirus interactions in our model described here. model proposed for bacterial or fungal pathogens

To encompass all the phenomena involved in the complicated arms race between a particular host and pathogen, the zigzag model is quite useful. The concept of the model may be applied also to host-virus interactions, paying attention to some analogous phenomena in PTI and ETI. For example, one review totally fit a model for host defense against viruses to the standard zigzag model, regarding viral dsRNAs as 
82 [12]. Consistent with this review, based on extreme resistance observed for tobacco

83 plants expressing the P19 protein, an RSS of tombusvirus, Sansregret et al. [13•]

84 showed that the general scheme of host induction and viral suppression of RNA

85 silencing could be adapted to the classical frame of PTI and ETI. However, in another

86 review, although some degree of analogy of PTI and ETI between viruses and other

87 pathogens was drawn, the author indicated a clear difference and the uniqueness in

88 virus-host interactions [11,14]. Fungal and bacterial pathogens have various Avr

89 proteins in their arsenal when ETI is activated; one can be replaced by other redundant

90 effectors. However, viruses have a limited number of proteins that are all important for

91 their survival. When one of the viral proteins is recognized by a host R protein, viruses

92 cannot easily replace it with another; rather they modify it by changing the amino acid

93 sequence while retaining the protein structure necessary for the function. Whether the

94 host R protein still recognizes the modified version depends on the LRR domain in the

$95 \mathrm{R}$ protein with a varying degree of affinity. Alternatively, according to the bait and

96 switch model, a host co-factor that binds to an R protein may affect the specificity of

97 the host recognition for the viral Avr protein [10]. Therefore, for virus-host

98 interactions, we cannot draw an actual zigzag model in which multiple rounds of ETS

99 followed by ETI are repeated with different combinations of host R protein and viral

100 Avr. As such, the molecular virus-host interaction must be explained by a limited

101 number of players.

102 Although the idea that RNA silencing and its suppression by viral RSSs can be

103 rationalized within the PTI-ETI framework is attractive, we need more experimental

104 evidence because there are actually viral proteins that are not RSSs but are recognized

105 by R-proteins. Instead of expanding on the standard zigzag model to fit virus-host

106 interactions, we can create a model that allows a quick overview of the molecular

107 phenomena in the virus-host arms race, the strategies unique to viruses and the steps

108 that are analogous to the standard zigzag model. As we will discuss, we consider that 
109 the host response branches from the general course of antiviral response instead of

110 repeating the ETI; the strategies at these branches vary depending on the specific host

111 and virus and the particular point of the interaction.

112

\section{RNA silencing and viral RNA silencing suppressors}

114 RNA silencing functions as an antiviral mechanism in plants [2,4] (Figure 1, B-D). As

115 a counterdefense, viruses developed RNA silencing suppressor (RSS) proteins, which

116 function to inhibit RNA silencing through diverse modes of action. The main

117 mechanism for the RSSs appears to be binding with long dsRNA or siRNA duplexes,

118 subsequently inhibiting siRNA biogenesis or RISC formation [15]. Another

119 mechanism is binding to the components in the silencing pathway such as AGO1.

120 Several RSSs (TCV CP, CMV 2b, TBSV P19, PVX P25, Polerovirus P0 and P1 of

121 Sweet potato mild mottle virus) have been reported to repress or interfere with the

122 function of AGO1 [16-21]. Diverse RSSs appear to reduce AGO1 in infected plant

123 tissues [22]. However, although viral RSSs interfere with host RNA silencing and are

124 mostly effective, hosts have some mechanisms to activate another or secondary

125 defense. For example, Arabidopsis thaliana encodes 10 AGO proteins. AGO1

126 performs not only antiviral RNA silencing, but also silences endogenous genes by

127 cleaving viral RNA and endogenous target mRNA. Recent screening of other AGO

128 proteins in antiviral defense using knockout mutants revealed that AGO2 is also

129 induced and functions in the defense against TCV and CMV when the viral RSSs

130 targeted AGO1 [23]. This study suggested that AGO2 is involved in antiviral RNA

131 silencing, which is induced via infection by viruses that encode RSSs targeting AGO1

132 or via miRNA-mediated RNA silencing because AGO2 expression is repressed by

133 AGO1 via miR403. In addition, miRNA-mediated RNA silencing appears to control

134 other RNA silencing components, including DCLs, DRB4, RDR6, and AGOs [24],

135 implying their involvement in secondary antiviral RNA silencing. Interestingly, AGO2 
136 is also involved in the induction and secretion of antimicrobial pathogenesis-related

137 protein 1, in addition to antiviral RNA silencing [25]. Since DRB4 is involved not only

138 in RNA silencing but also in R-gene-mediated resistance, if many R-genes are

139 controlled by miRNAs, seemingly, when a virus suppresses RNA silencing, diverse

140 secondary defense systems could be activated.

141

\section{Viral RNA silencing suppressors as Avr determinants}

143 Direct and indirect interactions occur between R-gene-mediated resistance and RSSs.

144 For example, a link between ETI-like phase and RNA silencing has been suggested for

145 Cucumber mosaic virus (CMV), Tobacco etch virus (TEV), and Potato virus Y (PVY).

146 The RSS of CMV, the 2b protein (CMV 2b), inhibits the salicylic acid (SA)-mediated

147 defense response [26]. Some examples of molecular interactions have been reported

148 between a viral RSS and an R-protein [27-29]. Well-established examples of host

149 recognition of an RSS are the coat protein (CP) of Turnip crinkle virus (TCV) and the

150 replicase of Tobacco mosaic virus (TMV). The TCV CP serves as an RSS, but also as

151 the TCV Avr protein that induces R-gene (the HRT gene)-mediated resistance in

152 Arabidopsis ecotype Di-17 [30,31]. TMV replicase has RSS activity [32], and the p50

153 helicase domain in the replicase can induce an HR, serving as the Avr determinant in

154 tobacco carrying the N-gene, which is the well-known R-gene working for ETI against

155 TMV.

156

157 Using an agroinfiltration assay to study the ability of viral RSSs to elicit HR-like

158 necrosis, Angel and coworkers [33] also found that the P19 protein (P19) of Tomato

159 bushy stunt virus (TBSV) was recognized by a putative R-protein in Nicotiana species,

160 which then induced an HR-like necrosis. Using a similar agroinfiltration assay for

161 Capsicum annuum, Ronde and colleagues [34] recently showed that the RSS of

162 Tomato spotted wilt virus (TSWV), the NSs protein, function as the Avr determinant in 
163 C. annuиm carrying the R-gene ( $T s w)$ against TSWV. They discussed their

164 pathosystem in light of the putative interplay between RNA silencing and the R-genemediated resistance.

167 Consistent with the reports by Angel and colleagues [33,35], P19 was recently

168 demonstrated to function as the Avr protein that induced extreme resistance (ER),

169 characterized by strong SA-dependent resistance without visible HR lesions, in

170 Nicotiana tabacum [13•]. In addition, the binding of P19 to small RNA (sRNA) was

171 necessary to induce ER, suggesting that RNA silencing and an ETI-like phase are

172 linked to each other. Similarly, the $2 \mathrm{~b}$ protein (TAV 2b) of Tomato aspermy virus

173 (TAV), an RSS of TAV, was found to induce HR on the leaves of N. tabacum and

174 Nicotiana benthamiana infected with the TMV vector expressing TAV 2b [36]. In this

175 case, Lys 21 and Arg 28, both located within the N-terminal region of TAV 2b, were

176 critical for the HR induction. These positively charged residues were later shown to be

177 involved in sRNA binding and thus the RSS activity of TAV $2 b$ [37]. These studies

178 thus suggest the existence of an R-protein that recognizes TAV $2 \mathrm{~b}$ with RSS activity as

179 an Avr protein in Nicotiana species. However, when its expression was driven by its

180 own parent virus or when $2 \mathrm{~b}$ of CMV, which is closely related to TAV, was expressed

181 by the TMV vector, no necrotic lesions were observed [36]. These contradictory

182 observations imply additional involvement of other viral or host factors in the HR-

183 associated resistance responses in specific combinations of RSS and host.

185 In further support of $2 \mathrm{~b}$ serving as an Avr protein, we recently demonstrated that CMV

$1862 \mathrm{~b}$ induced weak necrosis and SA and hydrogen peroxide accumulation in Arabidopsis

187 thaliana Col-0 ecotype (hereafter, Arabidopsis), suggesting that the plant has an R-

188 protein that recognizes CMV $2 \mathrm{~b}$ as an Avr protein. In fact, CMV Y strain (CMV-Y)

189 causes mosaics with fine necrotic spots in the upper leaves, but not typical HR-like 
190 necrosis, although we observed slightly stronger necrosis on Col-0 infected with CMV-

191 HL (a lily strain). From the results of an in situ molecular interaction study, the

192 necrosis on Arabidopsis seemed to have been driven by a specific interaction between

193 CMV 2b and the Arabidopsis catalase-3 (CAT3) [38••,39], a key enzyme in cellular

194 scavenging of hydrogen peroxide and induction of HR. If this type of HR-like

195 induction is indeed part of the host ETI-like phase, then an Arabidopsis R-protein may

196 recognize CMV $2 \mathrm{~b}$ as a complex with a host factor(s) that includes CAT3. The affinity

197 between CMV 2b and CAT3 seems to be important for determining the degree of

198 necrosis because the observed necrosis depends on the CMV strain and the

199 Arabidopsis ecotype.

200

201 miRNA-mediated regulation of the R-genes against viruses

202 Recent studies demonstrated that plant microRNAs (miRNAs) target and negatively

203 regulate R-gene expression via RNA silencing [29-33]. This miRNA-mediated R-gene

204 regulation was actually inhibited upon viral infection, suggesting that RNA silencing is

205 linked to R-protein-mediated resistance. Downregulation of R-gene expression by

206 RNA silencing is perhaps because plants prevent unwanted autoimmunity by

207 overexpressing the R-gene in the absence of viruses. Although RNA silencing

208 primarily targets viruses in the model, we also consider that RNA silencing can also

209 affect the subsequent host defense governed by R-proteins as discussed here.

211 Recent evidence has indicated that plant small RNAs (sRNAs) (siRNAs and miRNAs)

212 are involved in the basal resistance against pathogens. For bacteria, the bacterial

213 peptide flag22 actually induces miRNA393, which targets auxin receptors, which in

214 turn mediate the signaling that activates the SA resistance pathway [40]. In fact, an

215 RNA-induced silencing complex (RISC) containing Argonaute 2 (AGO2) programmed

216 with miR393 plays a critical role in ETI against Pseudomonas syringae [25]. The 
217 suppression of auxin signaling by miR393 ultimately activates the SA-mediated

218 defense response, which is also one of the main mechanisms in the antiviral PTI-like

219 phase; interference with the miR393-mediated regulation of auxin receptors by viral

220 suppressors must impair the PTI- and ETI-like phases against viruses.

221 Some sRNA can target R-gene transcripts directly. With the recent discovery of many

222 new miRNAs through deep-sequencing studies (e.g., RNASeq),. bioinformatics

223 analyses of the sRNA libraries obtained have identified novel miRNAs and putative

224 functions that potentially target host R-genes. For example, He and colleagues [41]

225 found an miRNA in Brassica rapa (named bra-miR1885) that was induced by Turnip

226 mosaic virus (TuMV) infection and potentially targeted the mRNAs of R-genes

227 encoding TIR-NB-LRR class proteins; unfortunately, whether the R-gene targets are

228 involved in the host resistance to TuMV was not determined. As another example,

229 after searching tobacco sRNA libraries for N-gene-related sRNAs, Li and coworkers

230 [42•] found that two newly discovered plant miRNAs (nta-miR6019 and nta-miR6020)

231 could guide cleavage of the N-gene transcript in tobacco, conferring resistance to TMV.

232 In a search for phased, trans-acting siRNAs (phasiRNAs) isolated from Medicago after

233 deep sequencing, Zhai and colleagues [43] revealed that the majority of phasiRNAs

234 were produced from R-genes, suggesting a close association between RNA silencing

235 and the R-gene-mediated resistance response. Although the phasiRNAs targets have

236 not been identified, we should consider generation of phasiRNAs when trying to

237 understand R-gene-mediated immunity.

238

239 Host factor(s) that regulate the interactions between RNA silencing and R-gene-

240 mediated resistance in a model for viruses

241 In our model for viruses (Figure 1B-D), viruses first produce dsRNAs in infected

242 plants. In turn, plants activate RNA silencing as a PTI-like phase to target the viral

243 RNAs. Then, the viruses produce RSSs as viral effectors to suppress RNA silencing. In 
244 the subsequent ETI-like phase, to generate an effective defense, the plants should

245 activate an R-protein that specifically recognizes the viral RSSs as the Avr protein,

246 thus leading to the HR and SA-dependent resistance. Although we have simplified

247 each phase to give a general overview, depending on the particular host-virus

248 combination, additional host factors should be integrated into the model for

249 understanding certain specific stages in host-virus interactions. For example, the RNA

250 silencing component DRB4 is potentially one such mediator of PTI-like phase. DRB4

251 is a dsRNA-binding protein that associates with a dicer-like protein 4 (DCL4) to

252 produce virus-specific siRNA [44,45]. A recent study revealed that the Arabidopsis R-

253 protein requires DRB4 for the subsequent HRT (an R protein to TCV)-mediated HR

254 against the RSS or CP of TCV [46••]. Notably, DRB4 interacts with both HRT and the

255 TCV CP and stabilizes HRT, but inhibits the interaction between HRT and the TCV

256 CP. Although we do not yet know how DRB4 contributes to the HR, we do know that

257 DRB4 is also involved in R-gene-mediated resistance against bacteria, implying that it

258 is involved in ETI. Another candidate mediator is the plant calmodulin-like protein,

259 rgs-CaM, which we describe in detail next.

260

261 Possible branches in the model between plants and viruses

262 RSSs that can suppress SA-related defense responses include CMV 2b, CaMV P6, and

263 TCV CP [14•,26,47-49]. Since viral RSSs participate in an arms race between viruses

264 and plants, and since RNA silencing is a PTI-like phase against diverse viruses, RSSs

265 reduce host defense, shifting the phase to effector-triggered susceptibility (ETS)-like in

266 the model. On the other hand, because some RSSs also behave as an SA-mediated

267 immunity suppressor (SIS), those RSSs can be considered to create another ETS-like

268 phase, implying additional branches in the model. All of these RSSs also function as

269 avirulence proteins, which can elicit the HR in plants that possess the corresponding R-

270 gene. In these cases, the HR is closely associated with SA-related defense responses. 
271 Integrating all these data, we propose a model in which viral SIS has been developed to

272 repress or evade the HR-mediated resistance against viruses (Figure 1, B and D). If a

273 virus has RSS or other viral proteins with SIS ability, SIS might be able to mask the R-

274 protein-mediated defense responses, resulting in a phenotype similar to that seen in a

275 susceptible plant. Therefore, we believe that many potential resistant interactions

276 between viruses and plants are still hidden. For example, the exacerbation of the HR

277 and symptoms that accompanies necrosis in plants infected with virus vectors that

278 express heterologous viral RSSs or other proteins [36,50] might be explained by the

279 induction of defense responses to the expressed viral proteins, which are not induced

280 by the parental viruses because of the viral SISs.

281

282 In addition to R-gene-mediated resistance, recent studies have suggested that plants

283 have additional counter-counterdefense systems against viral RSSs. We discovered an

284 antiviral counter-counterdefense that involves rgs-CaM in tobacco [51••,52]. When

285 rgs-CaM was initially found, rgs-CaM was reported to interact with the TEV RSS, HC-

286 Pro and act as an endogenous RSS [53,54]. Later, we found another function for rgs-

$287 \mathrm{CaM}$ in antiviral defense. Our previous study suggested that rgs-CaM binds not only

288 HC-Pro, but also other RSSs, including CMV and TAV 2b, via its affinity to the

289 negatively charged dsRNA-binding domains of RSSs. Then, rgs-CaM presumably

290 reinforces antiviral RNA silencing by directing the degradation of its associated RSSs

291 via autophagy (Figure 1, C and D). Calmodulin-like proteins are one of the three

292 protein families of EF-hand $\mathrm{Ca}^{2+}$ sensors in plants and are thought to coordinate the

293 functions of several endogenous proteins by binding to the targets as a hub protein in

294 response to the $\mathrm{Ca}^{2+}$ stimulus [55]. Since they are known to function in countering

295 abiotic and biotic stresses, we suspect that rgs-CaM functions in antiviral defense [41]. 296 
297 Recent studies on the interaction of the TCV CP with the Arabidopsis NAC

298 transcription factor (TIP) imply that an alternative branch should be included in the

299 model between viruses and plants. TCV CP is a viral RSS [56,57] and the avirulence

300 protein recognized by the R-gene, HRT in Arabidopsis Di-17 [31], suggesting that TIP

301 is involved in the RSS activity and HR induction by the TCV CP. However, TIP is not

302 required for either the RSS activity or HR induction. Instead, TIP was recently shown

303 to be involved in SA-mediated basal immunity in Arabidopsis [58]. TCV CP

304 suppresses the SA-mediated basal immunity via its binding to TIP [14•]. These studies

305 indicate that TCV CP suppresses both the RNA silencing and SA-mediated basal

306 immunity to facilitate the initial infection of Arabidopsis with TCV (Figure 1D). In the

307 canonical zigzag model for the interaction between other microorganisms and plants,

308 PTI should be an induced defense, which partly shares defense responses with ETI

309 after the perception of PAMPs by receptor-like kinases. Therefore, the TIP-associated

310 PTI seems to be integrated in the viral model as another interaction between virus and

311 host. Here, we draw those new interactions as branches in the viral model. More

312 interestingly, the basal resistance involving TIP also affects CMV accumulation,

313 indicating that the basal resistance is not specific to TCV [58]. For many other viruses,

314 similar sets of defense-related genes have been reported to be induced during viral

315 infection of a susceptible plant [59,60], suggesting that the host resistance response is

316 somehow suppressed in those plants, although it is partly activated. As such

317 interactions are uncovered, we can better organize the branches from the main course

318 of defense involving RNA silencing in the model for host-virus interactions.

320 Acknowledgements

321 This work was supported in part by Grants-in-Aid for Scientific Research from the

322 Ministry of Education, Culture, Sports, Science and Technology of Japan and the

323 Sumitomo Foundation. 
References and recommended reading

327 1. Ding SW: RNA-based antiviral immunity. Nat Rev Immunol 2010, 10:632-644.

328 2. Shimura H, Pantaleo V: Viral induction and suppression of RNA silencing in plants. Biochim Biophys Acta 2011, 1809:601-612.

330

3. Ding SW, Lu R: Virus-derived siRNAs and piRNAs in immunity and pathogenesis. Curr Opin Virol 2011, 1:533-544.

4. Burgyan J, Havelda Z: Viral suppressors of RNA silencing. Trends Plant Sci 2011, 16:265-272.

5. Giner A, Lopez-Moya JJ, Lakatos L: RNA silencing in plants and the role of viral suppressor. In: Martinez MA (ed) RNA interference and viruses. Caister Academic Press, Norfolk, UK, 2010, pp 25-46.

6. Wang MB, Masuta C, Smith NA, Shimura H: RNA silencing and plant viral diseases. Mol Plant Microbe Interact 2012, 25:1275-1285.

7. Jones JD, Dangl JL: The plant immune system. Nature 2006, 444:323-329.

- This is the first article on a zigzag model that explains PTI and ETI together.

8. Monaghan J, Zipfel C: Plant pattern recognition receptor complexes at the plasma membrane. Curr Opin Plant Biol 2012, 15:349-357.

9. Chinchilla D, Bauer Z, Regenass M, Boller T, Felix G: The Arabidopsis receptor kinase FLS2 binds flg22 and determines the specificity of flagellin perception. Plant Cell 2006, 18:465-476.

10. Mandadi KK, Scholthof KB: Plant immune responses against viruses: how does a virus cause disease? Plant Cell 2013, 25:1489-1505.

11. Moffett P: Mechanisms of recognition in dominant $\mathbf{R}$ gene mediated resistance. Adv Virus Res 2009, 75:1-33. 
12. Zvereva AS, Pooggin MM: Silencing and innate immunity in plant defense against viral and non-viral pathogens. Viruses 2012, 4:2578-2597.

13. Sansregret R, Dufour V, Langlois M, Daayf F, Dunoyer P, Voinnet O, Bouarab K: Extreme resistance as a host counter-counter defense against viral suppression of RNA silencing. PLoS Pathog 2013, 9:e1003435.

- This article is the first to demonstrate a direct link between RSS activity and the

Avr determinant for the ETI (R-protein-mediated resistance) in the zigzag model.

14. Donze T, Qu F, Twigg P, Morris TJ: Turnip crinkle virus coat protein inhibits the basal immune response to virus invasion in Arabidopsis by binding to the NAC transcription factor TIP. Virology 2014, 449:207-214.

\section{1}

- The Arabidopsis NAC transcription factor TIP is known to interact with the TCV $\mathrm{CP}$, which behaves as a viral RSS and avirulence protein in Arabidopsis Di-17 with the R-gene, HRT. Therefore, TIP was suspected of being involved in the RSS activity of TCV CP or its recognition by the R-gene, HRT. However, this study showed that in reality, TIP is involved in SA-mediated basal immunity and that TCV CP suppresses the basal immunity by binding to TIP.

15. Lakatos L, Csorba T, Pantaleo V, Chapman EJ, Carrington JC, Liu YP, Dolja VV, Calvino LF, Lopez-Moya JJ, Burgyan J: Small RNA binding is a common strategy to suppress RNA silencing by several viral suppressors. $E M B O J$ 2006, 25:2768-2780.

16. Derrien B, Baumberger N, Schepetilnikov M, Viotti C, De Cillia J, Ziegler-Graff V, Isono E, Schumacher K, Genschik P: Degradation of the antiviral component ARGONAUTE1 by the autophagy pathway. Proc Natl Acad Sci U S A 2012, 109:15942-15946.

17. Azevedo J, Garcia D, Pontier D, Ohnesorge S, Yu A, Garcia S, Braun L, Bergdoll M, Hakimi MA, Lagrange T, et al.: Argonaute quenching and global changes 
in Dicer homeostasis caused by a pathogen-encoded GW repeat protein. Genes Dev 2010, 24:904-915.

378

379

380

381

382

383

384

385

386

387

18. Giner A, Lakatos L, Garcia-Chapa M, Lopez-Moya JJ, Burgyan J: Viral protein inhibits RISC activity by argonaute binding through conserved WG/GW motifs. PLoS Pathog 2010, 6:e1000996.

19. Zhang X, Yuan YR, Pei Y, Lin SS, Tuschl T, Patel DJ, Chua NH: Cucumber mosaic virus-encoded 2b suppressor inhibits Arabidopsis Argonaute1 cleavage activity to counter plant defense. Genes Dev 2006, 20:3255-3268.

20. Chiu MH, Chen IH, Baulcombe DC, Tsai CH: The silencing suppressor P25 of Potato virus X interacts with Argonaute1 and mediates its degradation through the proteasome pathway. Mol Plant Pathol 2010, 11:641-649.

21. Varallyay E, Valoczi A, Agyi A, Burgyan J, Havelda Z: Plant virus-mediated induction of miR168 is associated with repression of ARGONAUTE1 accumulation. EMBO J 2010, 29:3507-3519.

22. Varallyay E, Havelda Z: Unrelated viral suppressors of RNA silencing mediate the control of ARGONAUTE1 level. Mol Plant Pathol 2013, 14:567-575.

23. Harvey JJ, Lewsey MG, Patel K, Westwood J, Heimstadt S, Carr JP, Baulcombe DC: An antiviral defense role of AGO2 in plants. PLoS One 2011, 6:e14639.

24. Qu F, Ye X, Morris TJ: Arabidopsis DRB4, AGO1, AGO7, and RDR6 participate in a DCL4-initiated antiviral RNA silencing pathway negatively regulated by DCL1. Proc Natl Acad Sci U S A 2008, 105:1473214737.

25. Zhang X, Zhao H, Gao S, Wang WC, Katiyar-Agarwal S, Huang HD, Raikhel N, Jin H: Arabidopsis Argonaute 2 regulates innate immunity via miRNA393*-mediated silencing of a Golgi-localized SNARE gene, MEMB12. Mol Cell 2011, 42:356-366. 
26. Ji LH, Ding SW: The suppressor of transgene RNA silencing encoded by Cucumber mosaic virus interferes with salicylic acid-mediated virus resistance. Mol Plant Microbe Interact 2001, 14:715-724.

27. Eggenberger AL, Hajimorad MR, Hill JH: Gain of virulence on Rsv1-genotype soybean by an avirulent Soybean mosaic virus requires concurrent mutations in both P3 and HC-Pro. Mol Plant Microbe Interact 2008, 21:931936.

28. Palanichelvam K, Cole AB, Shababi M, Schoelz JE: Agroinfiltration of Cauliflower mosaic virus gene VI elicits hypersensitive response in Nicotiana species. Mol Plant Microbe Interact 2000, 13:1275-1279.

29. Wen RH, Khatabi B, Ashfield T, Saghai Maroof MA, Hajimorad MR: The HCPro and $P 3$ cistrons of an avirulent Soybean mosaic virus are recognized by different resistance genes at the complex Rsv1 locus. Mol Plant Microbe Interact 2013, 26:203-215.

30. Choi CW, Qu F, Ren T, Ye X, Morris TJ: RNA silencing-suppressor function of Turnip crinkle virus coat protein cannot be attributed to its interaction with the Arabidopsis protein TIP. J Gen Virol 2004, 85:3415-3420.

31. Cooley MB, Pathirana S, Wu HJ, Kachroo P, Klessig DF: Members of the Arabidopsis HRT/RPP8 family of resistance genes confer resistance to both viral and oomycete pathogens. Plant Cell 2000, 12:663-676.

32. Wang LY, Lin SS, Hung TH, Li TK, Lin NC, Shen TL: Multiple domains of the Tobacco mosaic virus p126 protein can independently suppress local and systemic RNA silencing. Mol Plant Microbe Interact 2012, 25:648-657.

33. Angel CA, Hsieh YC, Schoelz JE: Comparative analysis of the capacity of tombusvirus P22 and P19 proteins to function as avirulence determinants in Nicotiana species. Mol Plant Microbe Interact 2011, 24:91-99. 
34. de Ronde D, Butterbach P, Lohuis D, Hedil M, van Lent JW, Kormelink R: Tsw gene-based resistance is triggered by a functional RNA silencing suppressor protein of the Tomato spotted wilt virus. Mol Plant Pathol 2013, 14:405-415.

35. Angel CA, Schoelz JE: A survey of resistance to Tomato bushy stunt virus in the genus Nicotiana reveals that the hypersensitive response is triggered by one of three different viral proteins. Mol Plant Microbe Interact 2013, 26:240-248.

36. Li HW, Lucy AP, Guo HS, Li WX, Ji LH, Wong SM, Ding SW: Strong host resistance targeted against a viral suppressor of the plant gene silencing defence mechanism. EMBO J 1999, 18:2683-2691.

37. Chen HY, Yang J, Lin C, Yuan YA: Structural basis for RNA-silencing suppression by Tomato aspermy virus protein 2 b. EMBO Rep 2008, 9:754760.

38. Inaba J, Kim BM, Shimura H, Masuta C: Virus-induced necrosis is a consequence of direct protein-protein interaction between a viral RNAsilencing suppressor and a host catalase. Plant Physiol 2011, 156:2026-2036.

-• CMV 2b was found to make a complex with Arabidopsis catalase-3 (CAT3) to induce necrosis in Arabidopsis plants. The degree of necrosis was correlated with the affinity between CMV $2 b$ and CAT3.

39. Masuta $\mathrm{C}$, Inaba J, Shimura $\mathrm{H}$ : The $2 \mathbf{b}$ proteins of Cucumber mosaic virus generally have the potential to differentially induce necrosis on Arabidopsis. Plant Signal Behav 2012, 7:43-45.

40. Navarro L, Dunoyer P, Jay F, Arnold B, Dharmasiri N, Estelle M, Voinnet O, Jones JD: A plant miRNA contributes to antibacterial resistance by repressing auxin signaling. Science 2006, 312:436-439. 
41. He XF, Fang YY, Feng L, Guo HS: Characterization of conserved and novel microRNAs and their targets, including a TuMV-induced TIR-NBS-LRR class R gene-derived novel miRNA in Brassica. FEBS Lett 2008, 582:24452452.

42. Li F, Pignatta D, Bendix C, Brunkard JO, Cohn MM, Tung J, Sun H, Kumar P, Baker B: MicroRNA regulation of plant innate immune receptors. Proc Natl Acad Sci U S A 2012, 109:1790-1795.

- This article shows that the overexpression of both nta-miR6019 and nta-miR6020 attenuated $\mathrm{N}$-mediated resistance to TMV, suggesting that those miRNAs are indeed functional.

43. Zhai J, Jeong DH, De Paoli E, Park S, Rosen BD, Li Y, Gonzalez AJ, Yan Z, Kitto SL, Grusak MA, et al.: MicroRNAs as master regulators of the plant NBLRR defense gene family via the production of phased, trans-acting siRNAs. Genes Dev 2011, 25:2540-2553.

44. Curtin SJ, Watson JM, Smith NA, Eamens AL, Blanchard CL, Waterhouse PM: The roles of plant dsRNA-binding proteins in RNAi-like pathways. FEBS Lett 2008, 582:2753-2760.

45. Fukudome A, Kanaya A, Egami M, Nakazawa Y, Hiraguri A, Moriyama H, Fukuhara T: Specific requirement of DRB4, a dsRNA-binding protein, for the in vitro dsRNA-cleaving activity of Arabidopsis Dicer-like 4. RNA 2011, 17:750-760.

46. Zhu S, Jeong RD, Lim GH, Yu K, Wang C, Chandra-Shekara AC, Navarre D, Klessig DF, Kachroo A, Kachroo P: Double-stranded RNA-binding protein 4 is required for resistance signaling against viral and bacterial pathogens. Cell Rep 2013, 4:1168-1184. 
$\bullet \quad$ This study revealed that the essential RNA silencing component DRB4 is also involved in R-protein-mediated HR responses, suggesting a link between viral PTI and ETI.

47. Lewsey MG, Murphy AM, Maclean D, Dalchau N, Westwood JH, Macaulay K, Bennett MH, Moulin M, Hanke DE, Powell G, et al.: Disruption of two defensive signaling pathways by a viral RNA silencing suppressor. $\mathrm{Mol}$ Plant Microbe Interact 2010, 23:835-845.

48. Laird J, McInally C, Carr C, Doddiah S, Yates G, Chrysanthou E, Khattab A, Love AJ, Geri C, Sadanandom A, et al.: Identification of the domains of cauliflower mosaic virus protein P6 responsible for suppression of RNA silencing and salicylic acid signalling. J Gen Virol 2013, 94:2777-2789.

49. Love AJ, Geri C, Laird J, Carr C, Yun BW, Loake GJ, Tada Y, Sadanandom A, Milner JJ: Cauliflower mosaic virus protein P6 inhibits signaling responses to salicylic acid and regulates innate immunity. PLoS One 2012, 7:e47535.

50. Hisa Y, Suzuki H, Atsumi G, Choi SH, Nakahara KS, Uyeda I: P3N-PIPO of Clover yellow vein virus exacerbates symptoms in pea infected with White clover mosaic virus and is implicated in viral synergism. Virology 2014, 449:200-206.

51. Nakahara KS, Masuta C, Yamada S, Shimura H, Kashihara Y, Wada TS, Meguro A, Goto K, Tadamura K, Sueda K, et al.: Tobacco calmodulin-like protein provides secondary defense by binding to and directing degradation of virus RNA silencing suppressors. Proc Natl Acad Sci U S A 2012, 109:1011310118.

•• This study revealed a novel counter-counterdefense system against viral RSSs in tobacco. In this system, the calmodulin-like protein rgs-CaM reinforces antiviral RNA silencing by directing the degradation of viral RSSs via autophagy. 
52. Tadamura K, Nakahara KS, Masuta C, Uyeda I: Wound-induced rgs-CaM gets ready for counterresponse to an early stage of viral infection. Plant Signal. Behav. 2012, 7.

53. Anandalakshmi R, Marathe R, Ge X, Herr JM, Jr., Mau C, Mallory A, Pruss G, Bowman L, Vance VB: A calmodulin-related protein that suppresses posttranscriptional gene silencing in plants. Science 2000, 290:142-144.

54. Nakamura H, Shin MR, Fukagawa T, Arita M, Mikami T, Kodama H: A tobacco calmodulin-related protein suppresses sense transgene-induced RNA silencing but not inverted repeat-induced RNA silencing. Plant Cell Tiss Org 2014, 116:47-53.

55. Bender KW, Snedden WA: Calmodulin-related proteins step out from the shadow of their namesake. Plant Physiol 2013, 163:486-495.

56. Deleris A, Gallego-Bartolome J, Bao J, Kasschau KD, Carrington JC, Voinnet O: Hierarchical action and inhibition of plant Dicer-like proteins in antiviral defense. Science 2006, 313:68-71.

57. Qu F, Ren T, Morris TJ: The coat protein of turnip crinkle virus suppresses posttranscriptional gene silencing at an early initiation step. $J$ Virol 2003, 77:511-522.

58. Jeong RD, Chandra-Shekara AC, Kachroo A, Klessig DF, Kachroo P: HRTmediated hypersensitive response and resistance to Turnip crinkle virus in Arabidopsis does not require the function of TIP, the presumed guardee protein. Mol Plant Microbe Interact 2008, 21:1316-1324.

59. Whitham SA, Yang C, Goodin MM: Global impact: elucidating plant responses to viral infection. Mol. Plant Microbe. Interact. 2006, 19:1207-1215.

60. Whitham SA, Quan S, Chang HS, Cooper B, Estes B, Zhu T, Wang X, Hou YM: Diverse RNA viruses elicit the expression of common sets of genes in susceptible Arabidopsis thaliana plants. Plant J. 2003, 33:271-283. 


\section{Figure legend}

\section{Figure 1}

536 The interactions between viral RSS (SIS) and host factors involved in plant immunity.

537 (A) Model of the arms race between pathogens and plants using the standard zigzag model. (B) Model of molecular virus-host interactions involving RNA silencing and R-protein (NB-LRR)-mediated resistance. Unlike the innate immunity against other

540 pathogens, the first layer of the immunity against viruses is RNA silencing. RNA

541 silencing is induced by intra- and intermolecularly formed double-stranded RNAs

542 (dsRNAs) of the viral genome or its transcripts. Then, dsRNA is processed into

543 siRNAs by the DCL4-DRB4 complex and DCL2 in Arabidopsis. AGO1 binds siRNA 544 and cleaves viral RNA guided by the incorporated siRNA. Most viruses counteract this

545 by expressing RNA silencing suppressors (RSSs). Plants coevolved an immune system

546 that is associated with the HR in response to the RSS. In this figure, the HR that is not 547 associated with SA-mediated resistance is defined as programmed cell death (PCD).

548 Recent studies have suggested that host cofactors such as DRB4, a tobacco

549 calmodulin-like protein, rgs-CaM, and the Arabidopsis NAC transcription factor (TIP)

550 help putative NB-LRRs to recognize RSSs [46••]. Salicylic-acid (SA)-mediated

551 defense responses were found to be suppressed by RSSs such as CMV $2 b$ and TCV CP,

552 suggesting viral evasion of induced HR, which is associated with the SA-mediated 553 immunity to prevent viral infection. Here, an SA-mediated immunity suppressor is 554 designated SIS. (C) Entire scheme to explain the host-virus interactions, integrating

555 steps unique to viruses compared with the standard zigzag model. (D) Branches from

556 the main path of the model, where viral factors (RSS and SIS) participate, represent

557 other virus-host interactions that are mediated by the same viral factors. For example,

558 the SA-mediated basal immunity involving TIP [58] and the rgs-CaM-directed 
559 degradation of RSS via autophagy [51••] are also thought to contribute to antiviral

560 immunity, although TIP and rgs-Cam seem to be independent of the general course of

561 host defense. TCV CP counteractively suppresses the basal immunity by binding to

562 TIP [14•].

563 

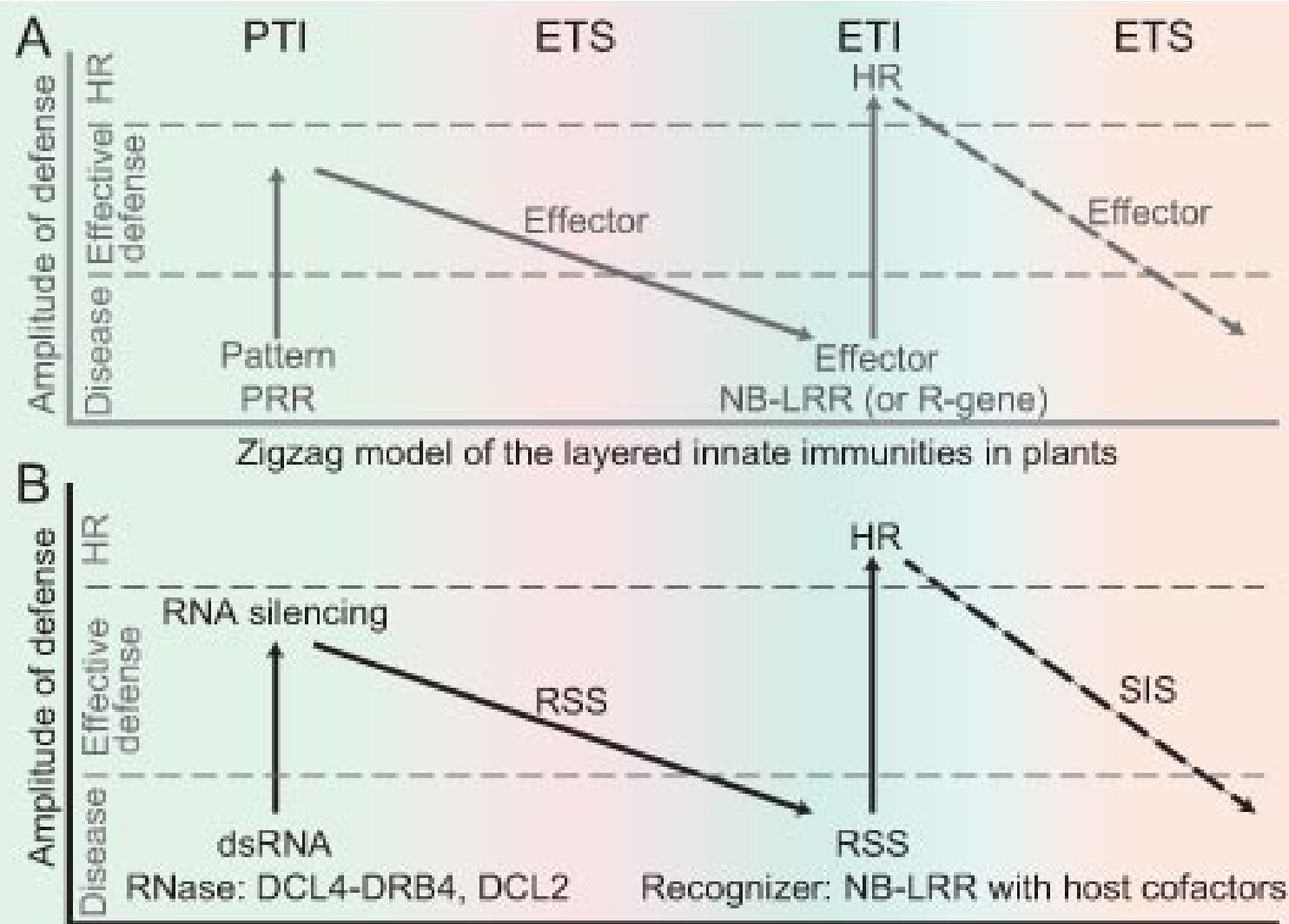

Main scheme of arms race between viruses and plants

C

Virus (dsRNA)—RNA silencing —viral RSS (SIS) — NB-LRR-mediated

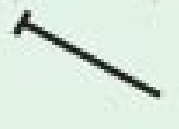

SA-mediated basal immunity
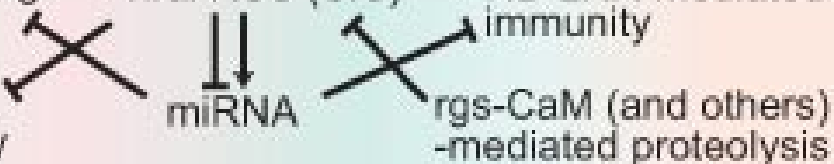

D

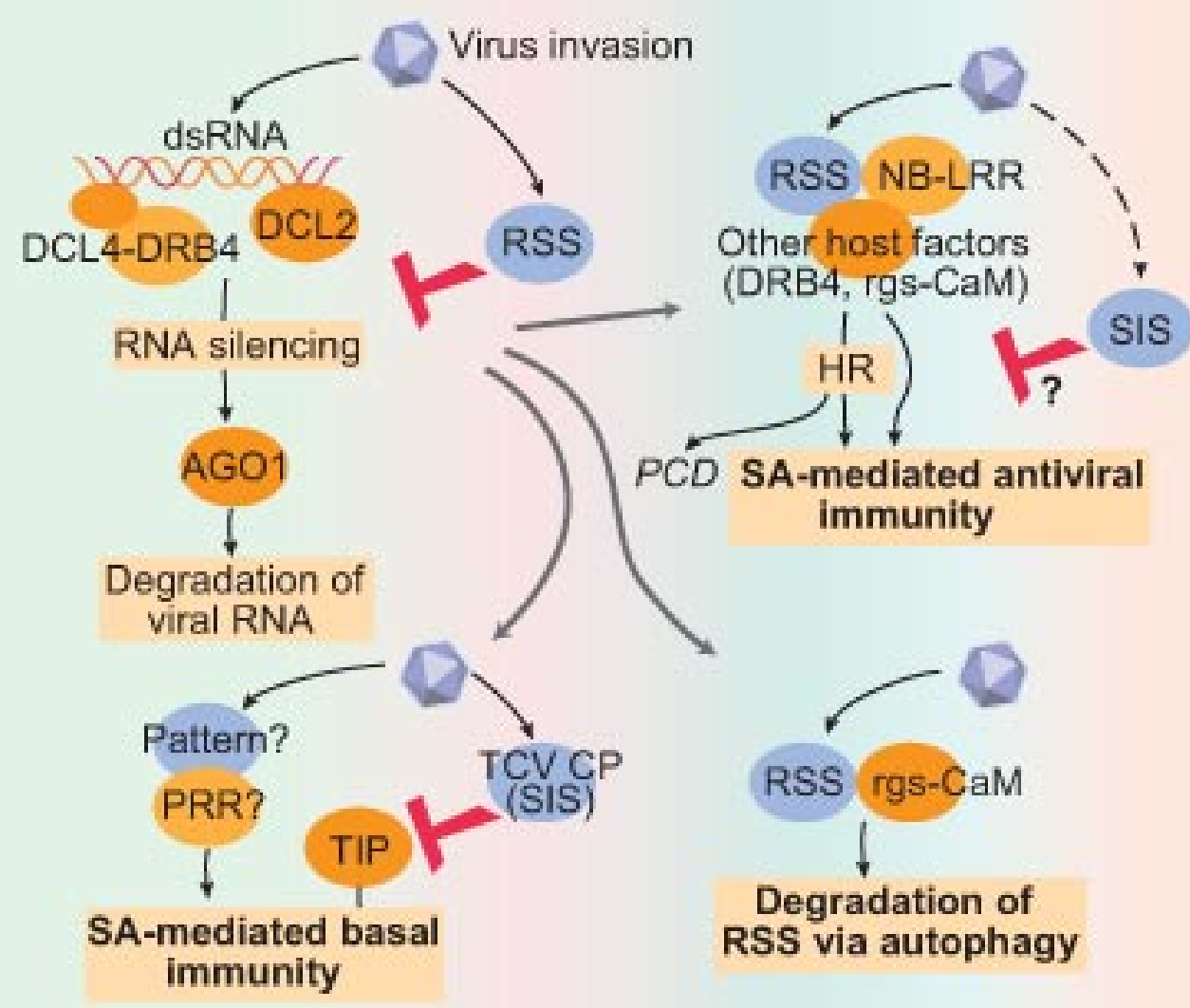

